\title{
Stefan Tomasek*
}

\section{Frauen- und Männerrollen in mittelhochdeutscher Kreuzzugslyrik}

DOI 10.1515/mial-2016-0008

\begin{abstract}
This article focuses on the effects that crusade motives woven into Middle High German courtly lovesongs have on gender binarism in these text. The analysis draws on two examples from the first period of Middle High German crusade poetry: Friedrich von Hausen, 'Si darf mich des zîhen niet' and Albrecht von Johansdorf, 'Guote liute, holt die gâbe'. I examine whether the relation between lady and male speaker is altered by the crusader's divine service and whether this affects the gender binarism in the songs. The article concludes with a discussion of the repercussions gender binarism has on the concepts of crusading in the two texts.
\end{abstract}

Keywords: German crusade poetry, Friedrich von Hausen, Albrecht von Johansdorf

Da im mittelhochdeutschen Minnesang in unterschiedlichen Rollen und Sprechsituationen stets Männer- und Frauenrollen aufeinander bezogen und so wechselseitig definiert werden, sind verschiedene Genderkonzepte zentral für das Genre. ${ }^{1}$ In der ab der Zeit des sog. Rheinischen Minnesangs (ca. 1170-1200) überlieferten mittelhochdeutschen Kreuzzugslyrik, ${ }^{2}$ um die es im Folgenden geht, ${ }^{3}$ wird das Abschiedsmotiv, das die mittelhochdeutschen Lieder seit ihren

1 Vgl. den Überblick bei Günther Schweikle, Minnesang. 2. Aufl. Stuttgart 1995, S. 169-195.

2 Alternativ ist in der mediävistischen Forschung der Terminus ,Kreuzlied' gebräuchlich. Dieser geht auf das mittelhochdeutsche kriuzliet zurück, das bei Reinmar dem Videler in einem vermeintlichen Gattungskatalog aufgeführt ist; vgl. Deutsche Liederdichter des 13. Jahrhunderts. Hrsg. v. Karl von Kraus. Bd. 1: Text. 2. Aufl. Tübingen 1978, 45,III. Da sich dieser Katalog aber als rhetorisches Spiel in einem Spottlied auf Leuthold von Seven findet und kriuzliet nicht definiert ist, erscheint der Terminus ,Kreuzzugslied` bzw. ,Kreuzzugslyrik‘ adäquater. Vgl. Stefan Tomasek, Minne, lâ mich vrî. Untersuchungen zur Kreuzzugslyrik des zwölften Jahrhunderts. Wiesbaden 2016, S. 19-22, zur Begriffsbestimmung.

3 Die Forschungssituation ist methodisch veraltet. Vgl. Friedrich-Wilhelm Wentzlaff-Eggebert, Kreuzzugsdichtung des Mittelalters. Studien zu ihrer geschichtlichen und dichterischen Wirklich-

*Kontakt: Dr. Stefan Tomasek, Lehrstuhl für deutsche Philologie, Ältere Abteilung, Universität Würzburg, Am Hubland, 97074 Würzburg, E-Mail: stefan.tomasek@germanistik.uni-wuerzburg.de 
Anfängen verwenden, mit Referenzstrukturen auf die zeitgenössischen Kreuzzüge kombiniert. Kreuzzugslyrik lässt sich somit als Variation der mittelhochdeutschen Abschiedslyrik beschreiben, durch die dem ansonsten stets unkonkret bleibenden oder nur aus der Minnesituation heraus begründeten Abschiedsmotiv ein historisch fassbarer Anlass eingeschrieben wird. Diese historische Referenz wird man nicht überbewerten dürfen: Auf welche historischen Ereignisse oder auch nur Phasen der Kreuzzugszeit in den Texten verwiesen wird, ist wegen der offenen Formulierungen fast nie $\mathrm{zu}$ entscheiden. ${ }^{4}$ Meist wird stattdessen auf das Motiv der Kreuzzug-conversio, wie im Falle Friedrichs von Hausen, oder auf Argumente der Kreuzzugswerbung, etwa bei Albrecht von Johansdorf, zurückgegriffen. Bezüge auf konkrete historische Entitäten finden sich in den mittelhochdeutschen lyrischen Kreuzzugstexten nur dann, wenn das zentrale Minnemotiv und damit die Diskussion bipolarer Genderkonzepte fehlt. ${ }^{5}$

keit, Berlin 1960; Hermann Ingebrand, Interpretationen zur Kreuzzugslyrik Friedrichs von Hausen, Albrechts von Johansdorf, Heinrichs von Rugge, Hartmanns von Aue und Walthers von der Vogelweide. Frankfurt a.M. 1966; Maria Böhmer, Untersuchungen zur mittelhochdeutschen Kreuzzugslyrik. Rom 1968; Ulrike Theiss, Die Kreuzlieder Albrechts von Johansdorf und die anderen Kreuzlieder aus ,Des Minnesangs Frühling‘. Freiburg 1974; Roswitha Wisniewski, Kreuzzugsdichtung. Idealität in der Wirklichkeit. Darmstadt 1984. Einschlägig daher noch immer: Peter Hölzle, Die Kreuzzüge in der okzitanischen und deutschen Lyrik des 12. Jahrhunderts. Das Gattungsproblem ,Kreuzlied‘ im historischen Kontext, 2 Bde. Göppingen 1980. Jüngere Publikationen vermögen dieses Desiderat einer methodisch fundierten Texterschließung nicht zu füllen; vgl. Ingrid Hartl, Das Feindbild der Kreuzzugslyrik. Das Aufeinandertreffen von Christen und Muslimen. Bern 2009; Lars Bültmann, Kreuzzugslyrik im hohen und späten Mittelalter. In: Jürgen Rauter (Hg.), Oswald von Wolkenstein. Literarische Tradition, Variation und Interpretation anhand ausgewählter Lieder. Rom 2009, S. 217-266. Vgl. auch Susanne Reichlin, Ästhetik der Inklusion. Inklusionsverfahren und Inklusionssemantiken in der mhd. Kreuzzugslyrik. Masch. Habilitationsschrift (im Erscheinen) und Tomasek (Anm. 2).

4 Vgl. Christa Ortmann u. Hedda Ragotzky, Das Kreuzlied. Minne und Kreuzfahrt. Albrecht von Johansdorf: ,Guote liute, holt die gâbe‘. In: Helmut Tervooren (Hg.), Gedichte und Interpretationen. Mittelalter. Stuttgart 1993, S. 169-190, hier S. 169: „Meist sind diese Bezüge auf Kreuzzugsrealität jedoch so allgemein, daß die Zuordnung der Lieder zu bestimmten Kreuzzügen und damit eine präzise Datierung schwierig ist“; vgl. grundsätzlich hierzu Tomasek (Anm. 2).

5 Einen Sonderfall stellt der ,Heiliggrableich“ (aus ,Des Minnesangs Frühling ‘ 96,1 ff., im Folgenden MF) Heinrichs von Rugge aufgrund seiner Gattungszugehörigkeit und Überlieferungsgeschichte dar, der den Tod Friedrichs I. explizit als aktuelles Thema verhandelt (vgl. v.a. Versikel IV-VII ${ }^{\mathrm{b}}$ ), so dass die Entstehungs- und Wirkungszeit dieses Textes sicher auf die zweite Jahreshälfte 1190 festlegbar ist. Hartmanns von Aue berühmte, allerdings in ihrem Bezug nicht eindeutige Saladinverse (MF 218,5 ff.: II,8 f.) könnten mit dessen Todesjahr (1193) einen terminus post quem darstellen, sind aber auch anders interpretierbar. Ansonsten finden sich vergleichbare historische Verweise nur bei den Liedern und Sprüchen Walthers von der Vogelweide und einigen weiteren Sangspruchdichtern (v.a. Bruder Wernher, Sigeher, Wilder Alexander), auch im Sangspruch dominiert klerikale Argumentation vor historischer Referenz. Einen Überblick über die 
Durch die Übernahme von Kreuzzugsmotiven werden in der Kreuzzugslyrik zwei hinsichtlich Wertigkeit und Fiktionalisierungsgrad höchst divergente Diskurse zueinander in Beziehung gesetzt, indem ein genuin klerikales Kreuzzugskonzept mit einem vor allem fiktional-literarischen Minnekonzept kombiniert wird. ${ }^{6}$ Diese Divergenz spielt textimmanent jedoch keine Rolle: Die Argumentation des Kreuzzugsdiskurses, deren klerikale Stichhaltigkeit in den Liedern nie zur Diskussion steht, wird in das System des Minnesangs integriert, innerhalb dessen die Plausibilität und der Absolutheitsanspruch der Minnebindung selbstverständlich sind.

Durch diese Diskursinterferenz bringen die Kreuzzugsmotive in die Kreuzzugslieder erstens das Potential ein, die durch die Minnesituation definierten Männer- und Frauenrollen neu zu akzentuieren. Definiert die Minnesituation bipolar „männliche und weibliche Geschlechterrollen [...] in ihrer spezifischen Interdependenz", ${ }^{7}$ tritt nun mit den Argumenten des Kreuzzugsdiskurses ein klerikal konnotierter Pol hinzu. Umgekehrt wirken zweitens aber auch die Gendervorstellungen der Minnesituation auf das normative Kreuzzugskonzept ein:

gender is a primary way of signifying relationships of power. It might be better to say, gender is a primary field within which or by means of which power is articulated. [...] Established as an objective set of references, concepts of gender structure perception and the concrete and symbolic organization of all social life. ${ }^{8}$

Und schließlich entsteht drittens aus diesem Wechselspiel eine dilemmatische Grundstruktur, da die Kreuzzugslieder für Frauen- und Kreuzzugsdienst keinen Dispens vorsehen, wodurch der Kreuzzugsabschiedssituation die Frage nach der Vereinbarkeit beider Dienste und damit beider Diskurse als Grundmotiv inhärent ist. $^{9}$

mittelhochdeutschen Texte (mit Auszügen aus der Epik u. a.) findet sich bei Ulrich Müller, Kreuzzugsdichtung. Tübingen 1979.

6 Vgl. Theiss (Anm. 3), S. 5: „[B]eide Themenkreise, Minnedienst und Kreuzzug, sind in den Kreuzliedern verknüpft“. Vgl. zudem Wisniewski (Anm. 3), S. 82: „[Die] Dichter verbinden in ihrer Kreuzzugslyrik das große Thema jener Zeit, die Minne und den Frauendienst, mit der Reflexion über den Dienst für Gott als Kreuzritter“. Vgl. ferner Hartl (Anm. 3), S. 51.

7 Dorothee Kimmich, Einleitung zu den Gender Studies. In: Dies., Rolf Günther Renner u. Bernd Stiegler (Hgg.), Texte zur Literaturtheorie der Gegenwart. Stuttgart 2003, S. 393-400, hier S. 394.

8 Joan Wallach Scott, Gender. A Useful Category of Historical Analysis. In: The American Historical Review 91 (1986), S. 1053-1075, hier S. 1069. Deutsche Übersetzung: Joan Wallach Scott, Gender. Eine nützliche Kategorie der historischen Analyse. In: Dorothee Kimmich, Rolf Günther Renner u. Bernd Stiegler (Hgg.), Texte zur Literaturtheorie der Gegenwart. Stuttgart 2003, S. 416440.

9 Die Kreuzzugslieder Hartmanns von Aue und Reinmars haben im Vergleich zu den übrigen Liedern der Rheinischen Zeit eine deutlich andere Struktur, da in ihnen das dilemmatische Grund- 
Aus dieser dreifachen Interdependenz wird, angelehnt an die bereits zitierten Überlegungen Joan Scotts, folgende Fragestellung abgeleitet: Erstens ist zu ermitteln, wodurch in den beiden Liedern Geschlechterrollen definiert werden und welche normativen Konzepte des Minne- und Kreuzzugsdiskurses diese Rollen „,in festgeschriebenen binären Gegensätzen [formen], in denen kategorisch [...] die Bedeutung des Mannes und der Frau, des Männlichen und des Weiblichen, festgestellt wird“. ${ }^{10}$ Zweitens gilt es zu klären, wie umgekehrt der Bezug von Frauen- und Männerrollen zum Kreuzzugsdiskurs definiert wird und welche Rollen- bzw. Sprecheridentität hieraus resultiert.

Als Beispiele werden zwei Lieder gewählt, die einen schwachen (Friedrich von Hausen) und einen gut fassbaren Kreuzzugsbezug aufweisen (Albrecht von Johansdorf) und damit die Kreuzzugsreferenz in den Rheinischen Liedern gut repräsentieren. Die genaue Datierung dieser Lieder in ihrem historischen Kontext lässt sich nicht genau fassen: Für Friedrichs von Hausen Lieder gibt es mit dessen Todesdatum (6. Mai 1190 auf dem Kreuzzug) immerhin einen terminus ante quem, eine Datierung auf die Zeit vor dem dritten Kreuzzug bleibt aber möglich; Albrechts von Johansdorf literarisches Schaffen lässt sich vor allem aufgrund mehrerer Generationen gleichlautender Namensträger nicht genau datieren.

\section{Friedrich von Hausen ,Si darf mich des zîhen niet}

Friedrichs von Hausen Lied ,Si darf mich des zîhen niet“ (MF 45,37 ff.) ${ }^{11}$ stellt eine für die erste Phase der deutschen Kreuzzugslyrik charakteristische Form mit

motiv zweier unauflösbarer Dienstverpflichtungen fehlt. Sie werden dementsprechend im Folgenden nicht dem Korpus Rheinischer Kreuzzugslyrik zugerechnet, ohne hiermit eine Aussage über ihre Entstehungszeit zu implizieren, da auch bei diesen Texten eine Datierung auf eine Zeit vor 1200 nicht auszuschließen ist.

10 Scott (Anm. 9), S. 431.

11 MF 45,37 ff. weist eine divergierende Überlieferungssituation auf, da es in HS B als vierstrophiges, in HS C jedoch als fünfstrophiges Lied verzeichnet ist, wobei die fünfte Strophe (MF 46,39 ff.) der C-Fassung in B als Einzelstrophe an späterer Stelle eingetragen ist. Hierbei zeigt sich diese Einzelstrophe jedoch nicht nur formal baugleich, sondern entspricht auch in der Grundsituation, Argumentation und den verwendeten Motiven exakt den vier Strophen aus MF 45,37ff. Im Folgenden wird daher von einem fünfstrophigen Lied ausgegangen. Aufgrund der problematischen Editionslage wird der Text im Folgenden zitiert nach Friedrich von Hausen, Lieder. Mittelhochdeutsch/Neuhochdeutsch. Text, Übersetzung und Kommentar. Hrsg. v. Günther Schweikle. Stuttgart 1984, S. 72-75, 82. Schweikle folgt bei seiner Texteinrichtung jedoch HS B als Leithandschrift und ediert dementsprechend als vierstrophige Fassung (Ton II) mit additiver Einzelstrophe (Ton V), im Folgenden als Strophe I-IV und Strophe V bezeichnet. 
schwach ausgeprägter Kreuzzugssituation dar: Ein conversio-Motiv (III,8) ${ }^{12}$ wird mit einem (hier nur angedeuteten) Abschiedsmotiv $\left(\right.$ II,5) ${ }^{13}$ verbunden. Diese Motivkombination ist die Grundkonstellation aller (Rheinischen) Kreuzzugslieder; im argumentativen Kern reduzieren sie den Kreuzzugsabschied auf das spirituelle conversio-Motiv. Dass gleichzeitig die Frauenrolle fehlt und nur aus der Perspektive des Mannes über eine Frau gesprochen wird, ist ebenso der Regelfall. Insofern ist Friedrichs Lied für die oben formulierte Fragestellung zwar im doppelten Sinne kein günstiges, aber ein typisches Beispiel der mittelhochdeutschen Lyrik dieser Zeit.

\section{Minne}

Die Minnesituation in Friedrichs von Hausen Lied ist einheitlich durch die Attribute einer klassischen Minneklage definiert. ${ }^{14}$ Diese resultieren jedoch nicht aus der Abschiedssituation, die kaum argumentativ genutzt wird, sondern aus dem ausbleibenden lôn (IV,2) der geliebten Dame.

Warum dem Sprecher die Gunst seiner Dame vorenthalten wird, ist im Text nicht erkennbar; dieser dienest âne lôn (IV,2: „Dienst ohne Lohn“) wird zwar durchaus als Makel verstanden (IV,3-5), ${ }^{15}$ führt aber keineswegs zur Abwertung der Geliebten: Si darf mich des zîhen niet, / ich enhete si von herzen liep (I,1f.). ${ }^{16}$ Im Gegenteil leitet der Sprecher das Lied damit ein und beteuert immer wieder, dass sein Minnedienst am aller beste $[n]$ wîp $(\mathrm{II}, 3)$ immer anhalten werde, wohin er auch gehe. ${ }^{17}$ Im Moment des Sprechens steht also die Dienstabsage trotz ausbleibenden Lohnes und Minneleid nicht zur Diskussion. Er sei ihr holt (II,6), konstatiert das Männer-Ich stattdessen, wobei holt „liebend“, aber auch „im Dienst stehend“ bedeuten kann und so den fortbestehenden Minnedienst mar-

12 Vgl. III,8: nû wil ich mich an got gehaben; IV,10: nû wil ich dienen dem der lônen kan; V,7-9: doch klage ich daz, / daz ich sô lange gotes vergaz, / den wil ich iemer vor in allen [allen frouwen] haben.

13 Vgl. II,5: swar ich iemer var.

14 Für den Text prägend ist die vom Sprecher beschriebene soziale nôt (I,5; IV,6), der strît / [...] / [...] wider daz aller beste wîp (II,1-3, „die Auseinandersetzung mit der allerbesten Frau“), grôze[] sorgen (III,1), klage (III,7), unmilte (IV,5, „Ungnade“), kumber (V,1) und schaden (V,3).

15 Vgl. IV,3-5: von der [vrouwe] sprich ich niht wan allez guot, / wan daz ir muot / wider mich ze unmilte ist gewesen - „von der spreche ich nichts außer alles Gute, nur dass ihre Einstellung mir gegenüber zu ungnädig gewesen ist“.

16 „Sie darf mich dessen nicht bezichtigen, dass ich sie nicht von Herzen lieb hätte“. So auch in IV,3-6.

17 Vgl. II,5: swar ich iemer var - „wohin auch immer ich mich begebe“. 
kiert. ${ }^{18}$ Die Unerfüllbarkeit der Liebe stellt aber keinen Selbstzweck dar, der Sprecher hatte es zunächst anders erwartet. ${ }^{19}$ Ungewöhnlich sei diese Minnesituation jedoch nicht: daz was diu minne, diu noch manigem tuot / die selben klage (III,6f.), ${ }^{20}$ konstatiert das Männer-Ich.

Damit ist in der Minnesituation dieses Liedes eine im Minnesang durchaus übliche hierarchische Struktur zwischen den Geschlechtern entwickelt, die sich durch den dienest des Mannes gegenüber der erhofften genâde der Frau auszeichnet. Diese hat die Entscheidungsgewalt über Erfüllung oder Nicht-Erfüllung der Liebe inne und auch die Zurückweisung wird als legitimes weibliches Verhalten akzeptiert. Damit kontrolliert sie uneingeschränkt die entscheidenden Parameter der Minnesituation. Diese Hierarchie wird textimmanent nicht weiter sozial begründet; außer der Vorrangstellung der Geliebten gegenüber allen anderen Frauen (II,3) und der Bezeichnung als sô rehte wolgetân (II,10: „wirklich von schöner Gestalt“), erfährt man nichts über die vrouwe des Sprechers. Ihre gegenüber dem Mann erhöhte soziale Stellung resultiert aus der Geschlechterkonzeption des Minnesystems selbst und ist, wie gesagt, unabhängig vom lôn-Motiv und insofern absolut gesetzt und nicht hinterfragbar. Der Mann hingegen muss sorge, klage, kumber und schaden sowie das ringen und den strît aushalten; die Unterstellung, der Sprecher hätte die Minnebindung aufgegeben, wäre sowohl seitens der geliebten Frau (I,1-4) als auch der übrigen Gesellschaft (V,1-6) ehrenrührig; der Sprecher verbittet sich dies. Im Gegenteil wäre das lâzen von diesem strît zwischen Mann und Frau für den Sprecher unsanfte (II,1f.: „bedrückend“) - der Not des Minnedienstes stünde also nur eine weitere Not gegenüber, Dispens ist keine Alternative. Dass die Liebe unter diesen Bedingungen ihrerseits soziales Störpotential birgt, da der Mann sô verre an si [die Dame] verdaht, so sehr in Gedanken an sie versunken gewesen sei, dass er den Gruß seiner Mitmenschen nicht mehr zur Kenntnis genommen habe $(\mathrm{I}, 10)$ und bisweilen zeitlich nicht mehr orientiert gewesen sei (I,6f.), wird als Beweis der anhaltenden Liebe nicht negativ gewertet, sondern ist Ausdruck der Herzensliebe. Auch insofern hat der Minnedienst also Vorrang vor anderen sozialen Strukturen - das Rollenmuster, das der Sprecher entwickelt, ist vollständig auf die vrouwe ausgerichtet und unterliegt den von der Dame kontrollierten Regeln.

18 Vgl. II,4: mîn lîp / muoz dienen - ,ich muss ihr dienen“.

19 Vgl. IV,6-9: vor aller nôt dô wânde ich sîn genesen, / dô sich verlie / mîn herze ûf genâde an sie, / der ich da leider funden niene hân - „Ich wähnte damals von aller Not erlöst zu sein, als sich mein Herz ihrer Gnade überließ, die ich dort leider nie gefunden habe“.

20 „Das war die Liebe, die noch immer manchem dasselbe Leid antut“. 


\section{Conversio}

Diese hierarchische Struktur sollte durch die Regeln der conversio hinsichtlich ihrer zentralen Punkte aufgehoben werden, indem nun Vorrangstellung und das Recht, zu lohnen, der vrouwe entzogen werden. ${ }^{21}$ Umgekehrt erscheint dadurch die (im Minnesystem fehlende) Möglichkeit, die soziale nôt aufzulösen, für den Sprecher realisierbar. ${ }^{22}$ An dieser Stelle wird also der oben formulierte dritte argumentative Pol in den Text eingebracht, der eine subversive Wirkung auf die bipolare Genderstruktur haben müsste.

Die ersten beiden Strophen, in denen von der conversio noch nicht gesprochen wird, zeigen bereits, dass es sich so einfach offenbar nicht verhält: Die Unterstellung der Dame, der Sprecher habe sich in seiner conversio von ihr abgekehrt (I,1f.), wird als nicht der wârheit $(\mathrm{I}, 3)$ entsprechend zurückgewiesen, das lâzen von der Minnenot als unsanfte (II,1f.) und der Dienst an der Dame als immerwährend (II,3-5) charakterisiert. Die conversio des Sprechers stellt also keine Abkehr von seiner eigenen weltlichen Minne dar, sondern erzeugt eine Interferenz von anhaltendem Damendienst und Gottesdienst. Sie wird dementsprechend auch nicht als Abkehr von der Geliebten beschrieben, sondern wendet sich gegen das allgemeingültige Konzept der Minne selbst: In der ersten conversio-Strophe (III) wird die vrouwe des Mannes nicht erwähnt, sondern nur von liebe (III,3) und minne (III,6; so auch V,1) als allgemeingültigem Prinzip gesprochen. ${ }^{23}$ Die Vorrangstellung Gottes wird als gegenüber allen Damen generell zutreffend ebenso kollektiviert ${ }^{24}$ wie das Lohnmotiv der dritten Strophe. ${ }^{25}$ Beides lenkt den Fokus von Dame und konkreter Liebessituation des Sprechers ab. Das sich an Gott Halten (III,8) wird als generell gültiges Prinzip beschrieben. Damit hat die Minne ein über die konkrete Liebessituation des Sprechers hinausgehendes Potential: Sie ist generell diskursiv wirksam und formt die normativen Geschlechterrollen trotz - oder besser: wegen - ihres sozialen Störpotentials einerseits und der Überschneidung mit der Kreuzzugsverpflichtung andererseits.

21 Vgl. IV,10: nû wil ich dienen dem der lônen kann -,Nun werde bzw. will ich dem dienen, der lohnen kann“; V,9: den [Gott] wil ich iemer vor in allen haben - „Den will ich immer vor ihnen allen in Ehren halten".

22 Vgl. III,9: der [Gott] kan den liuten helfen ûz der nôt - „der kann den Menschen aus der Not helfen".

23 Vgl. III,6f.: die noch manigen tuot / die selben klage.

$24 \mathrm{Vgl} \mathrm{V,6:} \mathrm{den} \mathrm{[Gott]} \mathrm{wil} \mathrm{ich} \mathrm{iemer} \mathrm{vor} \mathrm{in} \mathrm{[den} \mathrm{vrouwen]} \mathrm{allen} \mathrm{haben.}$

25 Vgl. III,9f.: der [Gott] kan den liuten helfen ûz der nôt. / nieman weiz, wie nâhe im ist der tôt „der kann den Menschen aus der Not helfen. Niemand weiß, wie nahe ihm der Tod ist“. 
Aufgehoben ist diese Technik der Generalisierung nur in der vierten Strophe, in der, durch eine Besonderheit der Endreimstruktur hervorgehoben, ${ }^{26}$ der fehlende lôn (IV,2) und die unterbliebene genâde (IV,8) der Dame als Motivation für die conversio des Sprechers angeführt werden. Bemerkenswert ist die geringe Aufmerksamkeit, die dem Lohn Gottes zukommt. Dies gilt bereits quantitativ: Nur einer von zehn Versen ist in dieser Strophe dem Gotteslohn gewidmet. Es gilt aber vor allem für die Erwartung des Sprechers, von aller Not errettet zu sein, indem er Herz und Seele der Gnade seiner Herrin überantwortet. ${ }^{27}$ Dies ist eine Formulierung, die der conversio gut anstünde und deutlich gegenüber den nüchternen Formulierungen nû wil ich mich an got gehaben (III,8) und nû wil ich dienen dem der lônen kan $(\mathrm{IV}, 10)$ hervorgehoben ist. Und auch dieser Strophe ist, wie gesagt, keine Dienstabsage inhärent; die Feststellung, ab sofort Gott zu dienen, konkurriert mit dem Postulat immerwährenden Dienstes an der Dame. ${ }^{28}$

Dass ein solches additives Dienstkonzept nicht unproblematisch ist, thematisiert der Text selbst: Die der Minnesituation anhaftende Gefahr, sich in Gedanken an die Dame vollständig zu verlieren, ${ }^{29}$ die in der Eingangsstrophe zunächst als soziales Phänomen beschrieben wird, korrespondiert, an gleicher Stelle im Strophenbau und auffallend parallel gestaltet, ${ }^{30}$ mit der Klage der Schlussstrophe, daz ich sô lange gotes vergaz $(\mathrm{V}, 8)$. Das eine ist die Folge aus dem anderen, ${ }^{31}$ das Motiv, sich selbst aufgrund der Liebe zu vergessen, bildet damit eine Klammer um alle Strophen.

26 Der erste Paarreim (I,1f.: undertân : nan) stellt aufgrund der vokalischen Länge eigentlich einen unreinen Reim dar, was an keiner anderen Stelle des Liedes der Fall ist. Das Reimpaar bildet aber mit dem ebenso unreinen Reim der beiden Schlussverse (hân : kan) einen reinen Reim, wodurch eine formale Klammer entsteht, die der Wiederaufnahme von Dienst- und Lohn-Motiv entspricht. IV,1: Dienst an der Dame vs. IV,10: Dienst an Gott; IV,2: fehlender Lohn der Dame vs. IV,9: fehlende Gnade der Dame.

27 Vgl. IV,6-8: vor aller nôt dô wânde ich sîn genesen, / dô sich verlie / mîn herze ûf genâde an sie.

28 Vgl. II,3-5: daz aller beste wîp, / der ie mîn lîp / muoz dienen.

29 Vgl. I,8: ich was sô verre an si verdâht.

30 Beide Verseingänge bilden zueinander einen identischen bzw. einen assonanten Halbreim als Chiasmus (I,8: ich was - V,8: daz ich); die adverbialen Bestimmungen sô verre (I,8) und sô lange $(\mathrm{V}, 8)$ bezeichnen in ihrer Grundbedeutung jeweils eine räumliche bzw. zeitliche Distanz, wenngleich verre hier als Demonstrativpronomen (so sehr) fungiert; nach der dritten metrischen Hebung steht in beiden Versen jeweils eine daktylische Struktur, die in den Strophen II-IV fehlt (bei Elision in IV,8); die Endreime beider Verse bilden wiederum Halbreime zu einander (I,8: verdâht : V,8: vergaz). Aus dieser parallelen Struktur fallen die Formulierungen an si (I,8) vs. gotes $(\mathrm{V}, 8)$ heraus, die sich so als Antagonismus gegenüberstehen.

31 Dies gilt auch insofern, als in V,1-6 die Minnesituation des Sprechers beschrieben wird, an die die Klage, Gott vergessen zu haben, adversativ angeschlossen wird: V,7: doch klage ich daz. 
Diese Klage des Sprechers ${ }^{32}$ war bereits in der Mittelstrophe (III), ebenfalls an gleicher Position, ${ }^{33}$ als minnetypisch eingeführt worden: Die Allgegenwärtigkeit der Liebe (vgl. III,4: daz verlie mich nie) habe dazu geführt, dass der Sprecher sich an wîsheit kêrte (III,5), von der Weisheit abgewendet habe. Sich auf die Liebe einzulassen, hat also im profanen wie im spirituellen Sinne ein destruktives Potential, es ist ein potentielles Abwenden von den legitimen sozialen Rollen (an wîsheit kêr[en]). Diesem kêren wird nun das an got gehaben (III,8) entgegengestellt, wodurch aber das liebevolle Denken an die vrouwe seine selbstverständliche Legitimität verliert ${ }^{34}$ und zur potentiellen Sünde wird. ${ }^{35}$

Die aus der conversio des Sprechers resultierende Interferenz von Frauenund Gottesdienst wird also in einer dreischrittigen Argumentation beschrieben: 1 . Der bipolaren Struktur des Minnesystems wird generell und in der konkret vorgeführten Minnesituation eine sowohl sozial als auch spirituell destruktive Wirkung zugeschrieben. 2. Dem unterbliebenen Minnelohn wird durch eine conversio der Lohn Gottes entgegengestellt. 3. Den Dienst an der Dame unter diesen conversio-Bedingungen fortzusetzen, ist illegitim, da es eine potentielle Sünde darstellt.

Dass die Liebe unter diesen Bedingungen dennoch persistiert und der Dienst für die Dame trotz fehlendem Lohn notwendigerweise anhält, unterläuft die conversio-Argumentation des Liedes. Hierin besteht gewissermaßen der Clou der Argumentation des Sprechers, der seinem durch die conversio entstandenen Dilemma ausweicht, indem er zwar der Minne generell eine Absage erteilt, aber sein Dienst an der konkreten Geliebten hiervon unberührt bleibt. Die Abwendung von der Minne wird nur im Lohnmotiv mit der eigenen vrouwe in Verbindung gebracht, ohne hierbei eine Wertigkeit zu Gunsten des Gotteslohnes zu formulieren. Da aber die fehlende Gunst der Dame diese ausdrücklich nicht diskreditiert (I,1f.; V,3-6), ist dies als additives Motiv zu verstehen. Die Leerstelle des Minnelohnes wird durch den Lohn Gottes gefüllt, ohne dass dies den dienest an der eigenen Herrin beeinträchtigt, da hier ja ohnehin kein Lohn möglich war. Die Minne generell wird ebenso wie der allgemeine Frauendienst ins zweite Glied gerückt, dies aber betrifft nicht die Bindung an die konkrete vrouwe.

Tatsächlich kann von der Vorrangstellung Gottes, wie es die Schlussstrophe in Hausens Lied formuliert, auch keine Rede sein: Dies gilt strukturell, da im Liedanfang in den ersten beiden Strophen ausschließlich die Minnebindung

32 Vgl. V,7: doch klage ich daz.

33 Vgl. III,7: die selbe[] klage.

34 Vgl. II,6f.: ich bin ir holt, swenne ich vor gote getar, / sô gedenke ich ir - ,ich liebe sie bzw. stehe in ihrem Dienst, wann immer ich es mich im Angesicht Gottes traue, denke ich an sie“.

35 Vgl. II,9: ob ich des sünde süle hân - „wenn ich hierbei Sünde haben sollte“. 
thematisiert und erst nachfolgend klerikale Argumente eingebracht werden. Und auch in den drei conversio-Strophen (III-V) ist der Strophenbeginn jeweils nur der Minne gewidmet - vor der klerikalen Argumentation hat formal im Gegenteil also stets die Minneargumentation Vorrang. Dies trifft auch quantitativ zu, da sich von den 50 Versen des Liedes nur sieben Verse mit Gott beschäftigen, obwohl der Sprecher dieser 50 Verse angibt, sich nun, also im Moment des Sprechens, an Gott zu halten (III,8), dies aber mit seiner eigenen Rede permanent unterläuft. Selbst in den drei conversio-Strophen hat die klerikale Argumentation keinen quantitativen Vorrang. ${ }^{36}$ Dies gilt auch in der argumentativen Struktur, indem auf die Ankündigung jeweils des Strophenschlusses, von nun an Gott zu dienen, nach dem Strophenwechsel stets erneut der Schwenk zurück auf die Geliebte erfolgt und betont wird, das Männer-Ich spreche über sie nur Gutes. ${ }^{37}$

Diese Gewichtung gilt aber vor allem hinsichtlich der conversio selbst: Die spirituelle Umkehr (convertere), die der Sprecher des Liedes für sich in Anspruch nimmt, entspricht keineswegs der zeitgenössischen Forderung einer Umkehr des Herzens. Die Mahnung, den Kreuzzug corde contrito, ${ }^{38}$ mit reumütigem Herzen zu führen, wie es Bernhard von Clairvaux und beispielsweise auch Gregor VIII. formulierten, ${ }^{39}$ entspräche in der Argumentation des Liedes einer Abkehr des Herzens von der Dame. Der Ich-Sprecher beschreibt aber die noch immer anhaltende liebe von herzen (I,2), das nur unsanfte lâzen des Herzens (II,1f.) und dessen verlâzen ûf genâde der Dame (IV,8). Auch der Umstand, dass bis zum letzten Vers das herze des Sprechers den vrouwen vorbehalten bleibt (V,10), während die Bindung an Gott nur mit den sehr nüchternen Verba gehaben („halten an“), dienen und haben („hochhalten“) beschrieben wird, zeigt, dass von einer vart corde contrito offenbar keine Rede sein kann. In den von hulde und persistierendem dienest geprägten gedanken an die Geliebte besteht die potentielle Sünde des Sprechers ebenso wie im Motiv des Sprechens über sie - damit, so ließe sich folgern, im Lied selbst, das in der Kreuzzugssituation nicht vom Thema der Minne ablassen kann.

36 III: drei conversio-Verse vs. sieben Minneverse; IV: 1 conversio-Vers vs. neun Minneverse; V: drei conversio-Verse vs. sieben Minneverse.

37 Vgl. IV,3: niht wan allez guot bzw. V,5: ir iht spreche wan guot.

38 Bernhard von Clairvaux, ,ep. 363,5`. In: Bernhardus Claraevallensis, Sämtliche Werke. Lateinisch/Deutsch. Hrsg. v. Gerhard B. Winkler. Bd. 3. Innsbruck 1992.

39 Vgl. ,Gregorii VIII papae, epistolae et privilegia‘. In: Patrologiae cursus completus, series Latina. Hrsg. v. J.-P. Migne. Bd. 202. Paris 1855, Sp. 1537-1564, hier Sp. 1542: qui corde contrito et humiliato spiritu itineris hujus laborem assumpserint. 


\section{Albrecht von Johansdorf, Guote liute, holt die gâbe'}

Während Friedrich von Hausen in ,Si darf mich des zîhen niet' die klerikale Argumentationsstruktur weitgehend zurückgenommen nur auf das Minnemotiv beschränkt, stehen zu Beginn und am Ende von Albrechts von Johansdorf ,Guote liute, holt die gâbe‘ (MF 94,15ff.) ${ }^{40}$ zwei Predigtstrophen mit Kreuzzugsbezug. Die klerikale Argumentation rahmt so zwei Minnestrophen, die ihrerseits als Wechsel gehalten sind, wobei die Frauenstrophe (III) durch eine inquit-Formulierung erneut formal abgesetzt erscheint. In beiden Predigtstrophen wird keine erkennbar eigene Sprechinstanz entwickelt, die Textautorität speist sich in ihrem prädikantischen Duktus ganz aus der Anlehnung an die Gattung der Kreuzzugspredigt. In I und IV spricht damit ein Prediger-Ich, das deutlich von den Sprechinstanzen der Minnestrophen getrennt erscheint, in denen aus der subjektiven Perspektive zweier Liebenden gesprochen wird. ${ }^{41}$ Die prädikantische Sprechsituation der Rahmenstrophen dient der Kontrastierung mit den beiden Minnesprechern, die Predigtstrophen schaffen den allgemeingültigen, normativen Kontext für die subjektive und ich-zentrierte Auseinandersetzung eines männlichen und einer weiblichen Sprecher-Rolle mit dem Kreuzzugsabschied. Dies unterstreicht nicht nur den Sprecherwechsel zwischen den beiden Männern (I und IV vs. II), sondern es verschiebt durch diesen Wandel vom Unpersönlich-Allgemeinen zum Subjektbezogen-Konkreten vor allem den Fokus des Liedes vom Kreuzzugsaufruf zum Minneabschied.

Die erste Predigtstrophe ist in ihrer Anrede an die [g] uoten liute (I,1) nicht genderspezifisch; das Märtyrermotiv wäre auf Männer- wie Frauenrollen übertragbar. Erst die Ausdifferenzierung der beiden Minnestrophen ${ }^{42}$ und die abschließende Predigtstrophe, die explizit weibliches Verhalten in Abgrenzung

40 Zitiert wird im Folgenden nach: Des Minnesangs Frühling. Bd. 1: Texte. Unter Benutzung der Ausgabe von Karl Lachmann und Moriz Haupt, Friedrich Vogt und Karl von Kraus. Bearb. von Hugo Moser und Helmut Tervooren. 38. Aufl. Stuttgart 1988. Der Text ist hier weitgehend nach der HS A wiedergegeben; in HS A steht dieses Lied jedoch unter dem Namen „Gedrut“. Da es sich bei Gedrut sehr wahrscheinlich um eine spätere Handschriftenbesitzerin gehandelt hat und der Text in HS C unter dem Namen Albrecht von Johansdorf überliefert ist, gilt die Autorzuweisung als unstrittig.

41 Beide Strophen enthalten sieben flektierte Verwendungen des Personalpronomens ich, wobei die drei Ansprachen (III,4-6) an den eigenen vröidelôse [n] lîp (III,4) in der Frauenstrophe letztlich ebenfalls Selbstansprachen darstellen.

42 II: Ein Männer-Ich reflektiert die reine[]gotes vart (II,5) in gotes lant (II,9); III: ein Frauen-Ich reflektiert die Trennung vom geliebten Mann. 
zum männlichen Kreuzfahrer definiert, erzeugen eine bipolare Struktur. Der prädikantische Eingang bildet den Bezugspunkt für die beiden Minnestrophen, die sich deutlich gegenüber der klerikalen Argumentation abgrenzen, während die dritte Strophe argumentativ unmittelbar mit der Schlussstrophe verzahnt ist.

\section{Klerikale Männerrolle}

Ebenso wie das conversio-Motiv im Lied Hausens steht auch die Eingangsstrophe ganz im Kontext von gâbe $(\mathrm{I}, 2)$ und solt $(\mathrm{I}, 4)$ Gottes einerseits und Dienstpflicht des Menschen für Gott andererseits. ${ }^{43}$ Während Lohn und Dienst bei Hausen aber abstrakte Begriffe bleiben, konkretisiert der Predigtsprecher der ersten Strophe in ,Guote liute, holt die gâbe‘ beides: Die vröiden iemer manecvalt (I,6: „die vielfachen, ewig währenden Freuden“), die er in Aussicht stellt, sind den vil saeldenhaften dort (I,5: „den sehr seligen dort“), d.h. also im Reich Gottes als iemer leben (I,10: „ewiges Leben“) versprochen, wenn sie den Dienst für Gott erfüllen. Dieser Dienst besteht in der Argumentation der ersten Strophe ausschließlich in Leidensund Opferbereitschaft: Lîdet eine wîle willeclîchen nôt (I,7) und gebt ime des lîbes tôt $(\mathrm{I}, 10)$, fordert das Prediger-Ich sein implizites Publikum mit dem Argument von Gabe und Gegengabe auf (I,9f.: got hât iu [...] gegeben. / gebt ime). Diese Zuspitzung auf das Märtyrermotiv, das den Tod als alleinigen Grund und zentrales Ziel der gotes vart definiert, sonst aber keinerlei militärische, politische oder ereignishistorische Argumente zulässt, dominiert die folgenden Strophen und wird im letzten Vers der Schlussstrophe erneut aufgenommen, in der die Reise über sê (IV,3) ebenfalls als ein aus der Welt Scheiden verstanden wird. ${ }^{44}$

Die Argumentation selbst entspricht zwar dem theologischen Gemeingut der Kreuzzugsrhetorik seit Urban II., sie unterschlägt aber einen wesentlichen Aspekt: Der für die theologische Flankierung des dritten Kreuzzugs verantwortliche Papst Gregor VIII. hatte in seiner Bulle ,Audita tremendi ${ }^{45}$ zwar durchaus in Aussicht gestellt, dass alle, die auf dem Kreuzzug unter Bereuung ihrer Sünden stürben, den vollständigen Sündenablass und das ewige Leben erlangen würden. Dies gelte jedoch auch unabhängig davon, ob man tatsächlich den Märtyrertod erleide oder den Kreuzzug überlebe. ${ }^{46}$ So predigte es etwas später nach dem

43 Vgl. I,4: dienent; I,7: Lîdet; I,10: gebt ime [Gott].

44 Vgl. IV,10: sich dirre welte $[. .$.$] bewegen.$

45 ,Gregorii VIII papae‘ (Anm. 40), Sp. 1539-1542.

46 Vgl. ,Gregorii VIII papae‘ (Anm. 40), Sp. 1542: qui corde contrito et humiliato spiritu itineris hujus laborem assumpserint et in poenitentia peccatorum decesserint, plenam suorum criminum 
Bericht Gunthers von Pairis auch Martin von Pairis vor dem vierten Kreuzzug in der Volkssprache. ${ }^{47}$ Es war also auch jenseits der päpstlichen Bullen opinio communis, dass, unabhängig von der Frage, ob man auf dem Kreuzzug den Märtyrertod starb oder überlebte, bereits die „Absolution im Bußsakrament [...] die Sündenschuld hinweg[nahm], die Kreuznahme [...] noch vor Antritt des Ablaßwerkes die Tilgung aller Sündenstrafen [bewirkte]“. ${ }^{48}$ Der Märtyrertod war also keineswegs die notwendige Bedingung für das iemer leben $(\mathrm{I}, 10)$, wie es die klerikale Argumentation der beiden Predigtstrophen nahelegt.

\section{Männlicher Minnesprecher}

Da in Albrechts Lied ebenso wie in allen anderen mittelhochdeutschen Kreuzzugsliedern die Argumente der Kreuzzugrhetorik nie infrage stehen, müssen sich die beiden Minnesprecher, die in den Mittelstrophen (II und III) das Grundmotiv des kreuzzugsbedingten Minneabschieds zum Thema machen, mit einem radikalen Trennungsmotiv auseinander setzen. Während es bei Hausen um die Wertigkeit und die (wenn auch problematisierte) Vereinbarkeit zweier Dienste geht, lässt die Märtyrer-Argumentation in ,Guote liute, holt die gâbe' letztere Option nicht zu. Der Dienst für Gott besteht im Opfertod auf dem Kreuzzug und schließt damit ein Fortbestehen jedweder anderer Dienstverpflichtungen kategorisch aus.

Die drei Anfangsverse der ersten Minnestrophe (II) fügen sich zunächst scheinbar in diese Argumentation ein und verwenden zudem die beiden zentralen Motive aus ,Si darf mich des zîhen niet“ (II,1-3): Minne, lâ mich vrî! / du solt mich

indulgentiam et vitam pollicemur aeternam. sive autem supervixerint, sive mortui fuerint, de omnibus peccatis suis, de quibus rectam confessionem fecerint, impositae satisfactionis relaxationem [...] se noverint habituros.

47 Vgl. Guntheri Cisterciensis, ,Historia captae a Latinis Constantinopoleos‘. In: Patrologiae cursus completus, series Latina. Hrsg. v. J.-P. Migne. Bd. 212. Paris 1865, Sp. 222-256, hier Sp. 228: Si autem quaeritis quid a Deo certi stipendii pro tanto labore sperare debeatis, certissime vobis polliceor quia quisquis signum crucis acceperit et puram fecerit confessionem, ab omni prorsus mundabitur peccato, et quocunque loco, vel tempore, seu casu praesentem reliquerit vitam, aeternam accipiet. Übersetzung nach Valmar Cramer, Die Kreuzzugspredigt zur Befreiung des Heiligen Landes 1095-1270. Köln 1939, S. 22: „Wenn ihr aber fragt, welch sicheren Lohn ihr von Gott erwarten dürft für so große Mühe, so hört, was ich euch mit aller Bestimmtheit verheiße: Wer das Zeichen des Kreuzes genommen und aufrichtig gebeichtet hat, soll alsbald rein sein von allen Sünden und das ewige Leben empfangen, gleichviel wo und wann und wie er das zeitliche verlieren wird“.

48 Hans Eberhard Mayer, Geschichte der Kreuzzüge. 3. Aufl. Stuttgart u.a. 1973, S. 45. 
eine wîle sunder liebe lân. / du hâst mir gar den sin benomen..$^{49}$ Der als Anrede an die personifizierte Liebe gehaltene innere Monolog greift die conversio-Aufforderung, das Entlassen aus dem Minnedienst, ebenso auf wie das Motiv des durch die Liebe verlorenen Verstandes. Ebenso wie bei Hausen stellt sich die Anforderung der Kreuzzug-conversio jedoch problematischer dar, als es zu erwarten wäre: zum einen, indem auch hier das $\hat{u} z$ mînem herzen scheiden (II,7) der Liebe als vil lîhte (II,8: „sehr wahrscheinlich“ oder „möglicherweise“) unmöglich beschrieben wird. Der Sprecher selbst hat hierauf keinen Einfluss, es obliegt allein der Minne selbst, ob sie ihn freilasse oder nicht. ${ }^{50}$ Unterbleibe dies, führe er sie mit sich in gotes lant (II,9), fährt der Sprecher fort, wodurch die oben für Hausen beschriebene Dienstinterferenz als Option im Raum steht, ohne problematisiert zu werden. Im Gegenteil integriert diese Variante eines gedoppelten Dienstes das Minnesystem unmittelbar in das Lohnkonzept des Kreuzzugs: Wenn die Minne ihn auf der gotes vart nicht freilasse, so beschließt das Männer-Ich die zweite Strophe, stünde der Geliebten der halbe Lohn zu. ${ }^{51}$ Hier ist also ein Ausgleichkonzept beschrieben, das, anders als bei Hausen, das Dilemma des Kreuzzugsabschieds vollständig auflöst. Die Formulierung, Gott werde umb halben lôn gemant (II,10), markiert den systemimmanenten Anspruch auf lôn, der aber aus einer doppelten konditionalen Struktur resultiert. Wenn die Minne das Herz des Mannes nicht freilasse, dann führe dieser sie mit sich nach Palästina und deswegen bestehe Anspruch auf hälftigen Lohn für die geliebte Frau. Dies steht in deutlichem Kontrast zum oben skizzierten skrupulösen Umgang mit dem Gedenken an die vrouwe bei Hausen.

Dies ist angesichts der Eingangsstrophe mit ihrem restriktiven Kreuzzugskonzept umso bemerkenswerter. Bereits der zweite Vers der ersten Minnestrophe verdeutlicht jedoch, dass die gotes vart des Minnesprechers keineswegs dem Märtyrerkonzept der Predigtstrophe entspricht: Diese wird als nur temporäre (II,2: eine wîle) Unterbrechung vom Minnedienst verstanden, nach deren Vollendung (II,5) - in der Argumentation der ersten Strophe bedeutete dies den Opfertod - die Rückkehr in den profanen Minnedienst in Aussicht steht. ${ }^{52}$ Das Leidmotiv (I,7f.) fehlt dementsprechend in der zweiten Strophe, göttlicher Lohn besteht nicht im unmittelbaren Erreichen des Gottesreiches durch den Märtyrertod des Mannes

49 „Minne, lass mich frei! Du sollst mich eine Weile ohne Liebe sein lassen. Du hast mir vollständig den Verstand geraubt“.

50 Vgl. II,7: [w]ilt aber dû [die Minne] ûz mînem herzen scheiden niht.

51 Vgl. II,9f.: vüere ich dich danne mit mir in gotes lant. / sô sî er der guoten dort umb halben lôn gemant - „dann führe ich dich mit mir in Gottes Land. Dann sei er dort an den halben Lohn für die Gute gemahnt“.

52 II,6: sô wîs [Anrede an die Minne]mir aber willekomen - „dann sei mir abermals willkommen“. 
(I,10), denn dies wäre nicht teilbar, sondern im Sündenablass für Mann und Frau. ${ }^{53}$ Diese Argumentation entspricht der oben skizzierten zeitgenössischen klerikalen Position und wäre im historischen Kreuzzugsdiskurs gut verankert. Sie widerspricht aber explizit dem Kreuzzugskonzept der Eingangsstrophe; die gotes vart des Minnesprechers fällt aus dem normativen Konzept des textimmanenten Predigers heraus.

Dieser Effekt stellt sich ein, da der Minnesprecher der zweiten Strophe zwar die gotes vart (II,5) in gotes lant (II,9) benennt, aber, der Verwendung des conversio-Motivs bei Hausen vergleichbar, tatsächlich nicht vom Kreuzzug spricht. Nur diese beiden Verse beziehen sich überhaupt auf ihn, ohne hierbei über das Attribut hinaus, diese Fahrt sei reine, also „ohne Sünde“, klerikale Aspekte einzubringen. Damit bleibt die Kreuzzugsargumentation der ersten Strophe zwar scheinbar unwidersprochen, aber sie kann sich im Minnesystem der zweiten Strophe nicht weiter entfalten und wird, da in ihrer zentralen Aussage unterlaufen, letztlich subversiv gebrochen. Die gotes vart erscheint so als Minnefahrt, deren Argumente um die Frage von Rückkehr zum profanen Minnedienst oder dessen Persistieren angesiedelt sind. Dass ausgerechnet das zentrale lôn-Motiv der ersten Strophe für das Männer-Ich nicht einmal eine Erwähnung wert zu sein scheint, sondern von diesem ausschließlich auf die guote (II,10) bezogen wird, inkorporiert auch dieses Argument in das Minnesystem. Der Minnesprecher reflektiert damit weder die Argumente der Kreuzzugspredigt in der ersten Strophe noch erfüllt er die diskursiv vorgegebene conversio-Anforderung, seine gotes vart corde contrito anzugehen. ${ }^{54}$ Das Herz bleibt unwendic, unumkehrbar, also nahezu wörtlich der conversio entgegengestellt, der Minne überlassen, die allein über das scheiden zu bestimmen vermag.

Die Männerrolle in ,Guote liute, holt die gâbe‘ ist daher ausschließlich über die Minnebindung definiert. Ähnlich wie bei Friedrich von Hausen überlagert die Liebe die ratio des Mannes und kann auch unter den Bedingungen der Kreuzzugconversio nicht überwunden werden. Sie entfaltet jedoch nicht das gleiche problematische Potential, sondern integriert die Kreuzzugssituation ganz in das System der Minne. Letzteres ist, gerade im Vergleich mit dem conversio-Motiv bei Hausen, aus drei Gründen bemerkenswert:

Erstens wäre zu erwarten, dass das deutlich stärker herausgestellte und an explizit klerikalen Normen ausgerichtete Märtyrermotiv ebenso wie der prädikan-

53 Hierzu fügt sich auch eine Variante der Überlieferung: HS A überliefert den letzten Vers der zweiten Strophe abweichend von der hier zitierten HS C (sô sî er der guoten dort) so: Sô sî der guoten hie er umbe halben lôn gemant. Damit bleibt diese überlieferungsgeschichtlich ältere Fassung ganz dem weltlichen Kontext (hie) des göttlichen Lohnes verhaftet.

54 Vgl. Anm. $38 f$. 
tische Duktus der Eingangsstrophe zu einer klerikal definierten Männerrolle führt. Das normativ-klerikale Konzept der Predigtstrophe dient aber zur subversiven Abgrenzung der Männerrolle in der zweiten Strophe.

Zweitens sollte die viel konkreter gestaltete und benannte Kreuzzugssituation die fiktionalen Minnemotive beeinflussen. Auch hier markiert der hoch ästhetisierte Sprechmodus des inneren Monologes an die personifizierte Minne den Unterschied zum an zeitgenössische Kreuzzugspredigten angelehnten Duktus der Predigtstrophe. Analog hierzu entstammen auch die Argumente der zweiten Strophe dem fiktionalen Minnesystem, der diskursive Kreuzzugshintergrund spielt nahezu keine Rolle, das Lohnmotiv wird in das Minnesystem integriert.

Drittens fehlt, anders als bei Hausen, die Beschreibung der konkreten Minnesituation, durch die das Sprecherverhalten begründet wäre. In der Anrede an die Liebe selbst wird hier ausschließlich das generell subversive Potential der Minne an einem Beispiel vorgeführt.

Diese Allgemeingültigkeit der Minneregeln steht im Kontrast zu den allgemeingültigen Normen der Predigtstrophe, ohne dass dieses Spannungspotential in der zweiten Strophe thematisiert würde. Ähnlich der Struktur in ,Si darf mich des zîhen niet' werden hierdurch die normativen Ansprüche des Kreuzzugsdiskurses unterlaufen; während Hausens Männer-Ich dies aber immerhin noch als potentielle Sünde markiert, nennt der Minnesprecher in ,Guote liute, holt die gâbe‘ sein Konzept zweier miteinander kombinierter Dienste explizit reine, sündenfrei, obwohl der Kontrast zum klerikalen Konzept textimmanent viel deutlicher gegeben ist.

\section{Weiblicher Minnesprecher}

Einen entscheidenden Unterschied gegenüber den meisten Kreuzzugsliedern Rheinischer Zeit stellt die dritte Strophe in ,Guote liute, holt die gâbe dar, da sich hier eine Frauenstimme zum Kreuzzugsabschied des Mannes zu Wort meldet. ${ }^{55}$ Auffällig ist die Hierarchisierung der Sprechebenen mittels einleitender inquitFormulierung und indirekter Rede. Indem nicht ersichtlich wird, auf welcher

55 Die Lieder Friedrichs von Hausen weisen keinerlei Frauenrede auf. Bei Albrecht von Johansdorf findet sie sich nur noch in einer (leider verderbten) Strophe $(87,5$, II) - erhalten ist ein Satz und fragmentierte indirekte Rede. Ferner steht eine Frauenstrophe im Wechsel Ottos von Botenlauben. Zudem sind acht Verse von dreihundert in den sog. Gespielinnenversikeln (X und $X^{b}$ ) im ,Heiliggrableich“ Heinrichs von Rugge in weiblicher Rede gehalten. Das Primat männlichen Sprechens ist allerdings kein Spezifikum der Kreuzzugslyrik, sondern gilt im Minnesang generell; vgl. Schweikle (Anm. 1), S. 181-195. 
Sprechebene diese Einleitung angesiedelt ist, indem ferner nur von einer Frau (III,1: ein wîp), nicht aber von der guoten (II,10) des männlichen Minnesprechers die Rede ist, die unmittelbar zuvor eingeführt worden war, handelt es sich offenbar nicht um eine konkrete Instanz der Minnesituation des Textes, sondern um allgemeines weibliches Sprechen. Dazu wird in der Schlussstrophe durch ein idealtypisches weibliches Sprechen ein Gegenentwurf entwickelt.

Der Sprechduktus der dritten Strophe entspricht dem inneren Monolog der zweiten Strophe, in der anstelle der personifizierten Minne allerdings der eigene vröidelôse [...] lîp (III,4) angesprochen wird. Den irdischen lîp hatte bereits die erste Predigtstrophe in der Dichotomie sêle vs. lîp als zu überwinden vorgeführt, dies jedoch unmittelbar folgend rhetorisch gebrochen: Die Aufforderung zum Märtyrertod führt gerade nicht das zu erwartende Konzept („der Tod des Körpers führt zum ewigen Leben der Seele“) fort, sondern verwendet das Lexem lîp in einer distinctio ${ }^{56}$ gedoppelt (,gebt ihm den Tod des Körpers [lîp], das wird für euch [dem lîbe] zum ewigen Leben werden“). ${ }^{57}$ Auch wenn dieses Wortspiel, das den im vorhergehenden Vers gerade erst eingeführten Dualismus von Körper und Seele scheinbar wieder aufhebt, aufgrund des textuellen Kontextes zunächst etwas überraschend erscheint, ist diese distinctio (lîp bedeutet sowohl „Körper“ als auch die „persona“ insgesamt) weder sprachhistorisch ${ }^{58}$ noch im zeitgenössischen literarischen Kontext ${ }^{59}$ besonders ungewöhnlich und daher leicht aufzulösen. Dennoch ist durch die rhetorische Struktur des Strophenschlusses die Funktion des Körpers besonders betont, wodurch die Wiederaufnahme in der Frauenstrophe, die letztlich das ambivalente Wortspiel fortsetzt, ${ }^{60}$ den Rückbezug zum Märtyrermotiv der Eingangsstrophe herstellt.

56 Vgl. Heinrich Lausberg, Handbuch der literarischen Rhetorik. Eine Grundlegung der Literaturwissenschaft. 4. Aufl. Stuttgart 2008, S. 333-335, § 660-662.

57 Vgl. I,10: gebt ime des lîbes tôt, daz wirt deme lîbe ein iemer leben. Diese Variante findet sich allerdings nur in HS A, HS C setzt die Dichotomie lîp vs. sêle fort.

58 Vgl. zum Lexem lîp als Bezeichnung für die Person seit althochdeutscher bzw. frühmittelhochdeutscher Zeit die Belege bei Helene Adolf, Wortgeschichtliche Studien zum Leib/Seele-Problem. Mittelhochdeutsch lip „Leib“ und die Bezeichnung für corpus. Wien 1937, S. 47-51, hier S. 49: „Die Dichter der Blütezeit haben also nur die alte Tradition fortgesetzt“. Vgl. ferner auch Deutsches Wörterbuch. Hrsg. v. Jacob und Wilhelm Grimm. Bd. 12. Leipzig 1885, Sp. 582f.

59 Vgl. die Beispiele in: Mittelhochdeutsches Handwörterbuch. Hrsg. v. Matthias Lexer. Bd. 1. Leipzig 1872, Sp. 1930-1932; Mittelhochdeutsches Wörterbuch. Mit Benutzung des Nachlasses von Georg Friedrich Benecke Hrsg. v. Wilhelm Müller und Friedrich Zarncke. Bd. 1. Leipzig 1854, Sp. 102-104,5.

60 In der Anrede an den eigenen Körper ist lîp zunächst wörtlich zu verstehen; da es sich hierdurch aber letztlich um ein Selbstgespräch handelt, fungiert lîp zugleich als Synekdoche und steht für die persona insgesamt. 
Die Abschiedsklage der Frau verhält sich gegenüber der ersten Predigtstrophe bemerkenswert ablehnend. Während die männliche Minnestrophe die Predigtanforderungen einfach ignoriert, führt das weibliche Pendant Leid und Trauer aufgrund des Minneabschieds vor; die dritte Strophe erscheint hierbei wie eine Antwort auf die erste:

Während im zweiten Vers der ersten Strophe den Märtyrern die gâbe Gottes verheißen wird (I,2), steht an gleicher Position in der dritten Strophe das leit der Liebe (III,2). Dem solt Gottes nach dem Tod (I,4) entspricht die (physische) Trauer des vröidelôse $[n]$ Körpers (III,4) der Dame. Den Märtyrern verheißt der Prediger der Eingangsstrophe, dass sie als die vil saeldehaften ins Reich Gottes (dort) eingehen (I,4f.), der zurückgebliebenen Frau droht aber der Verlust der sozialen Orientierung im Diesseits durch das hinnen varn des Mannes (III,4f.), ${ }^{61}$ wobei hinnen varn sowohl den $\mathrm{Abschied}^{62}$ als auch den $\operatorname{Tod}^{63}$ bedeuten kann. Während die Predigt mit den vröiden des ewigen Lebens $(\mathrm{I}, 6)$ argumentiert, beklagt die Frau den Verlust der einstigen frohen Gesinnung (III,6: hôchgemuot).

Dem Dualismus der göttlichen Schöpfung (I,9) ${ }^{64}$ entspricht ein Dualismus des Leidens (III,9). ${ }^{65}$ Der Prediger der ersten Strophe verspricht seinem impliziten Publikum, dass ihr tôt als Märtyrer zum iemer leben führe (I,10), der Frau bleibt aber nur die nôt aufgrund des hinnen varn (Abschied oder Sterben) ihres Geliebten (III,10), wobei die Lexeme tôt und nôt an gleicher metrischer Position strophenübergreifend einen reinen Reim bilden und so miteinander korrespondieren. Am deutlichsten tritt der Antagonismus zwischen den beiden Strophen in der (formalen) Wiederaufnahme des Märtyrermotivs zu Tage: Die Kadenzen der beiden Schlussverse der ersten Strophe (I,9f.: gegeben : leben) werden als identische Reime in der dritten Strophe wiederholt (III,7 f.: geleben : gegeben), aber in der Reimstellung verkehrt. Dies entspricht der konträren Argumentationsstruktur der jeweiligen Verspaare: Gebt Gott euren Tod, dies führt zum ewigen Leben (I,9f.) vs. das diesseitige Weiterleben steht durch das Leid über den Verlust des Geliebten infrage (III,7f.). Die Frauenstrophe bildet also einen formalen und argumentativen Antagonismus zur einleitenden Predigtargumentation.

Dieser Antagonismus entsteht jedoch, vergleichbar der zweiten Strophe, ohne dass die klerikalen Normen explizit Erwähnung finden. Auch die Frauenrolle des Liedes wird also zunächst ausschließlich im System der Minnebindung

61 Vgl. III,4f.: vröidelôser lîp,/wie wil du dich gebâren, swenne er hinnen vert.

62 So wird das Trennungsmotiv in II,4f. verwendet.

63 So wird das Trennungsmotiv in I,4 f. verwendet.

64 Vgl. I,9: got hât iu beide sêle und lîp gegeben.

65 Vgl. III,9: kund ich mich beidenthalben bewarn - „könnte ich mich auf beiden Seiten (d.h. vor dem freudlosen Weiterleben in der Welt und der eigenen Trauer) schützen“. 
definiert: Der Abschied des geliebten Mannes führt zur vollständigen sozialen Dissoziation (III,7), die nicht eigenständig vom Frauen-Ich zu überwinden ist, sondern der Unterweisung bedürfte: dâ bedorft ich râtes zuo gegeben. ${ }^{66}$ Dass der Konjunktiv dieser Formulierung verdeutlicht, dass der Appell der Eingangsstrophe offenbar nicht den rât darstellt, den es zur Lösung der Notsituation bedürfte, passt hierbei zur konträren Argumentation der Frau und bereitet gleichzeitig die abschließende Predigtstrophe vor. Zunächst aber steht das sehr präsente und stark betonte Minneleid ${ }^{67}$ im Mittelpunkt der Strophe, durch das Minne und der geliebte Mann zum einzigen Lebensmittelpunkt stilisiert werden. Die Anforderung des Kreuzzugsaufbruchs können dementsprechend nur die soziale Frauenrolle destruieren, ein Ausweichkonzept, wie in der zweiten Strophe vorgestellt, gibt es in dieser Perspektive nicht. Hinzu kommt, dass in der Ansprache an den eigenen Körper die physische Präsenz stark betont wird. Für den Anspruch des Beieinanderseins der Körper, das dem Liebeskonzept der Frauenstrophe zugrunde liegt, kann weder das Märtyrerkonzept der Eingangsstrophe noch das abstrakte Minnekonzept der zweiten Strophe eine Lösung bieten. Dementsprechend stellt die Frauenstrophe eine negative Antwort auf beide Kreuzzugskonzepte dar: Das hinnen varn (,sterben“) der Predigtstrophe führt genauso wie das hinnen varn („Abschied nehmen“) der zweiten Strophe zur körperlichen Trennung, aus der in jedem Fall Leid und soziale Not resultiert. Insofern passt es, dass das Abschiedsmotiv in seiner ambivalenten Formulierung (hinnen varn) als Polyptoton zweimal, genau in der Strophenmitte und im Strophenschluss, aufgenommen wird - beiden Formen des Abschieds, Märtyrertod oder diesseitige gotes vart, ist das gleiche entscheidende Manko inhärent.

Albrechts Frauenklage stellt damit einen Kontrapunkt zu zwei abstrakt verbleibenden Konzepten dar, indem sowohl der normative klerikale Anspruch der Predigt als auch das artifizielle Gedankenspiel der Minneanrede am konkreten Beispiel der Frauenstrophe im Leid der zurückbleibenden Frau scheitern. Es sind damit zwei (in sich bereits konträre) Männerrollen beschrieben, die aber beide dem Anspruch der Frau nicht genügen. Damit vermag auch das Ausgleichkonzept der Männerrolle nur solange eine Lösung zu implizieren, wie es bloße Minne-Theorie bleibt. Tauglich für die tatsächliche Liebe zwischen Mann und Frau und damit für den zentralen sozialen Aspekt der beiden Mittelstrophen ist es gerade nicht, da nur die Minne, aber eben nicht die Geliebte im Herzen mitzuführen ist.

66 „dazu bedürfte ich unterweisender Hilfe“.

67 Vgl. III,1: Ôwê; III,2: leit; III,3: leit; III,4: vröidelôs; III,6: du waere ie hôchgemuot; III,7: klage; III,8: „der Wunsch, sich vor dem Leid schützen zu können“; III,10: nôt. 


\section{Klerikale Frauenrolle}

Auf dieses Dilemma antwortet nun die letzte Predigtstrophe, die eine Synthese aus dem Leidmotiv der ersten und dem des Mitführens in gotes lant der zweiten Strophe anbietet. Hier wird die wîbes güete (IV,2) als sozialer Wert (IV,5: den sol er loben) eingeführt, die im Motiv des Mitleidens in der Heimat bestehe. Dieses Mitleiden im Gedenken an das Leiden und den Märtyrertod des Geliebten ${ }^{68}$ sei dem Leid der Kreuzfahrer vergleichbar. ${ }^{69}$ Dieses Motiv des Mitleidens, durch das die im Abendland verbliebenen Frauen dennoch am Kreuzzug partizipieren, passt vor allem zur Kreuzzugsargumentation Innozenz' III. und hat insofern einen diskursiven Hintergrund in der Kreuzzugsrhetorik des dreizehnten Jahrhunderts. ${ }^{70}$ Diese Argumentation passt ebenso zur Leid- und Märtyrerargumentation der ersten Predigtstrophe. Sie fügt sich aber nicht zu beiden Minnestrophen.

Denn die Männerstrophe (II) kennt, wie gesagt, weder das Leidmotiv noch die Vorstellung einer gotes vart als aus der Welt scheiden (IV,10: sich dirre welte [...] bewegen), sondern will räumliche Distanz im Gegenteil durch das Weiterbestehen der Liebe und das Zurückkehren in die Heimat überwinden. Die in der Minnestrophe (II) angelegte Männerrolle fügt sich dementsprechend nicht zum prototypischen Männerideal der vierten Strophe, mit dem ein Mitleiden aus der Heimat möglich wäre, wodurch aber die argumentative Basis der Schlussstrophe infrage gestellt ist. Das der zweiten Predigtstrophe zugrundeliegende Männerbild ist lediglich kohärent zur idealtypischen Männerrolle der ersten Strophe, gegenüber der sich aber, wie gesagt, der Männersprecher der zweiten Strophe ebenfalls absetzt.

Der engen Verzahnung zwischen erster und dritter Strophe vergleichbar, greift auch die vierte Strophe auf zentrale Motive der Frauenstrophe (III) zurück: Die Schlussstrophe nimmt das lip-Motiv zweimal (IV,4; IV,10) auf, wobei hier die Trennung in den guoten lîp der Frau (IV,4) und den aus der Welt scheidenden süeze[n] lîp $(\mathrm{IV}, 10)$ des Mannes genau das Leidmotiv der Frauenstrophe beschreibt. Hierbei steht das conversio- bzw. Märtyrer-Motiv (IV,9f.) ${ }^{71}$ an gleicher Strophenposition wie das Märtyrer-Motiv der ersten Strophe (I,9f.). Wiederum bilden die zentralen Verba der Kadenzen (IV,9f.: pflegen : bewegen) hierbei

68 IV,7f.: Swenne sî gedenket an sîne nôt./,lebt mîn herzeliep oder ist er tôt [...]' - „wenn sie an seine Not denkt: ,Lebt meine Herzensliebe noch oder ist sie tot?““

69 IV,6: wande ir heime tuot alsô wê - „denn ihr Daheimbleiben tut ebenso weh“.

70 Vgl. Michael Menzel, Kreuzzugsideologie unter Innocenz III. In: Historisches Jahrbuch 120 (2000), S. 39-79. Vgl. den Beitrag von Harald Wolter-von dem Knesebeck in diesem Heft.

71 IV,9f.: sô müeze sîn pflegen, / dur den er [...] sich dirre welte hât bewegen. - „so möge derjenige sich seiner annehmen, um dessentwillen er diese Welt aufgegeben hat“. 
assonante Halbreime zu den entsprechenden Versen der ersten und dritten Strophe (I,9f: gegeben : leben; III,7f.: geleben : gegeben) und sind so in die Verzahnung der Reimstrukturen eingegliedert, wodurch auch hier der Vergleich mit den entsprechenden Versen der ersten und dritten Strophe nahegelegt wird. Dies führt vor, wie wenig auch das Ausgleichskonzept der Schlussstrophe geeignet ist, das Problem der Frauenstrophe zu lösen: Die Vorstellung eines abstrakten vüere [n] über sê (IV,3) ist inkongruent zum konkreten Anspruch des physischen Beieinanderseins der Körper (III,4f.). Das verklärte Leidmotiv (IV,1: Wol si, saelic wîp), das als wîbes güete (IV,2) zur generellen weiblichen Norm stilisiert wird, hat keinerlei Gegenpart in den vielfachen Leidausrufen der Frauenstrophe. Die Apostrophierung der leidenden Frau als vil guote[r] lip $(\mathrm{IV}, 4)$ durch den Prediger steht (wiederum an gleicher Strophenposition) auffallend diskrepant zur Selbstanrede der Frauenrolle an den eigenen vröidelôse [n] lîp (III,4).

Obwohl die Schlussstrophe also eine Synthese der zentralen Motive der ersten drei Strophen anbietet (I: Leid- und Märtyrermotiv; II und III: das Motiv des Mitführens nach Palästina; III: Motiv der Körperlichkeit), verfehlt sie den Anspruch beider Minnerollen: Für die weltliche Männerrolle ermöglicht das Fortbestehen der Minne, nicht das Leiden des klerikalen Märtyrerkonzeptes, ein Überwinden der aus dem Kreuzzugsaufbruch resultierenden räumlichen Distanz; der Trennungsschmerz der weltlichen Frauenrolle resultiert aus dem konkreten körperlichen Verlust des Geliebten, der durch das abstrakte gedenken (IV,7) an das Leid des Mannes nicht zu mindern ist.

\section{Fazit}

In Friedrichs von Hausen ,Si darf mich des zîhen niet‘ wird eine hierarchische Geschlechterkonstellation inszeniert, in der der Mann trotz des sozial und klerikal destruktiven Potentials der Liebe einerseits und des fehlenden Minnelohns andererseits an seiner Minnebindung festhält, obwohl mit dem göttlichen Lohn die Alternative zur weltlichen Liebe im Raum steht. Damit bleibt die Männerrolle auch in der conversio-Situation durch die weltliche Bindung an die Frau definiert; die bipolare Geschlechterstruktur verändert sich durch das conversio-Motiv letztlich nicht. Das Ausgleichskonzept eines additiven Dienstverhältnisses ist im zeitgenössischen diskursiven Kontext und im Text selbst als problematisch markiert, aber für den Sprecher alternativlos. Insofern wirkt die Minne subversiv auf das klerikale Konzept, indem sie die im Text selbst postulierte Vorrangstellung des Gottesdienstes infrage stellt. Das conversio-Motiv nobilitiert letztlich nur die Treue zur geliebten Frau, die Männer- (und Frauenrolle) bleibt ausschließlich durch die Minnebindung definiert. 
Männer- und Frauenrolle in ,Guote liute, holt die gâbe‘ sind zwar im Vergleich dazu vor dem Hintergrund konkreter ausformulierter Kreuzzugswerbung inszeniert, aber der Text kommt zum gleichen Schluss. Die idealtypischen Männer- (I) und Frauenrollen (IV) der Kreuzzugspredigt passen nicht zu den durch die Minne definierten Genderentwürfen (IIf.). Dies ist umso bemerkenswerter, als der Kreuzzug selbst, der von den Minnesprechern nicht infrage gestellt wird, für die Frauenrolle als destruktiver Eingriff in die eigene soziale Verankerung verstanden wird, während das Kreuzzugskonzept des Mannes die zentralen Anforderungen der Predigt negiert. Während Hausens Minnesprecher die individuelle Unmöglichkeit vorführt, sich aus der Minnebindung zu lösen, zeigt die Diskrepanz zwischen den vom Kreuzzug betroffenen Sprechern und der idealtypischen Predigt bei Albrecht das Auseinanderfallen von klerikaler Kreuzzugstheorie und profanen Männer- und Frauenrollen. Der dritte Pol des klerikalen Kreuzzugsdiskurses vermag es also ebenso wenig, die durch die Minne definierte bipolare Geschlechterstruktur zu durchbrechen, indem die gotes vart zur Minnefahrt verkehrt oder ihre sozial destruktive Folge vorgeführt wird.

Beide Texte beantworten letztlich mit unterschiedlichen Techniken die drei eingangs gestellten Fragen auf die gleiche Weise: Erstens bleibt es auch unter den Bedingungen des Kreuzzuges bzw. der conversio stets dem diskursiven System der Minne vorbehalten, Männer- und Frauenrollen zu definieren; der klerikale Kreuzzugsdiskurs dient nur dem Kontrast. Zweitens wird das Kreuzzugskonzept, obwohl textimmanent nie infrage gestellt, implizit problematisiert, da es sich nicht in die profanen Ansprüche des dominanten Minnediskurses einfügt. Drittens skizzieren beide Texte damit Sprecheridentitäten, die, zwischen zwei Diskursen angesiedelt, nach Alternativ- und Ausgleichskonzepten suchen bzw. an diesem Dilemma zu scheitern drohen. Der Kreuzzug erscheint so als destruktiver und destabilisierender Faktor innerhalb der durch die Minne geprägten Geschlechterordnung beider Texte. 